\title{
Pathogen-induced changes in floral scent may increase honeybee- mediated dispersal of Erwinia amylovora
}

\author{
Antonio Cellini $\oplus^{1} \cdot$ Valentino Giacomuzzi ${ }^{2}$ Irene Donati ${ }^{1}$ Brian Farneti ${ }^{3}$ - Maria T. Rodriguez-Estrada ${ }^{1}$ \\ Stefano Savioli ${ }^{1} \cdot$ Sergio Angeli $\mathbb{i}^{2} \cdot$ Francesco Spinelli $^{1}$
}

Received: 13 June 2018 / Revised: 31 October 2018 / Accepted: 6 November 2018 / Published online: 30 November 2018

(c) International Society for Microbial Ecology 2018

\begin{abstract}
Honeybees are well recognised for their key role in plant reproduction as pollinators. On the other hand, their activity may vector some pathogens, such as the bacterium Erwinia amylovora, the causative agent of fire blight disease in pomaceous plants. In this research, we evaluated whether honeybees are able to discriminate between healthy and E. amylovora-infected flowers, thus altering the dispersal of the pathogen. For this reason, honeybees were previously trained to forage either on inoculated or healthy (control) apple flower. After the training, the two honeybee groups were equally exposed to inoculated and control flowering apple plants. To assess their preference, three independent methods were used: (1) direct count of visiting bees per time frame; (2) incidence on apple flowers of a marker bacterium (Pantoea agglomerans, strain P10c) carried by foragers; (3) quantification of E. amylovora populations in the collected pollen loads, proportional to the number of visits to infected flowers. The results show that both honeybee groups preferred control flowers over inoculated ones. The characterisation of volatile compounds released by flowers revealed a different emission of several bioactive compounds, providing an explanation for honeybee preference. As an unexpected ecological consequence, the influence of infection on floral scent increasing the visit rate on healthy flowers may promote a secondary bacterial spread.
\end{abstract}

\section{Introduction}

Pollinating insects, such as honeybees (Apis mellifera L.), are a crucial part of ecosystems for their contribution to plant reproduction. This role is also prominent in commercial orchards, since production, fruit growth and fruit durability are affected by pollination and seed development [1]. Honeybees regulate their foraging activity with the

Electronic supplementary material The online version of this article (https://doi.org/10.1038/s41396-018-0319-2) contains supplementary material, which is available to authorized users.

Francesco Spinelli

francesco.spinelli3@unibo.it

1 Department of Agricultural and Food Sciences, Alma Mater Studiorum - University of Bologna, viale G. Fanin 44, Bologna 40127, Italy

2 Faculty of Science and Technology, Free University of BozenBolzano, Piazza Università 5, Bolzano 39100, Italy

3 Department of Genomics and Biology of Fruit Crops, Research and Innovation Centre, Fondazione Edmund Mach, Via E. Mach 1, S. Michele all'Adige (TN) 38010, Italy perception of sensory cues, such as electric fields [2], chromatic/UV and symmetry patterns [3], flower temperature patterns [4] and volatile organic compounds (VOCs) $[5,6]$. In addition, they show a floral constancy, restricting foraging to one or a few flowering species at a given time, based on the memory of reward/flying distance ratio and the communication among members of the colony [7].

During their foraging activity, pollinators may vector plant pathogens [8]. Some of these pathogens manipulate insect behaviour to enhance their own spread, primarily by modifying host scent and/or nutritional reward [9]. However, the role of VOCs in pollinator-mediated pathogen transmission has been investigated only for a few, highly specialised fungal species: pseudoflower-producing foliar pathogens, such as Puccinia spp., attract insects to promote their own spread or sexual conjugation [10-12], while the infection by anther pathogen Microbotryum violaceum, associated to lower nutritional reward and VOC release, reduces the visitation rate by oviposing moths [13].

In comparison to fungal and viral pathogens, reports of bacteria vectored by pollinators are few, and only relating to the genera Erwinia and Pseudomonas [8, 14]. Among such pathogens, E. amylovora (Burrill) is the best studied and 
most destructive, being responsible for the fire blight disease in pomaceous plants [15]. The primary infection occurs through flowers [16-18]. Subsequently, E. amylovora can colonise systemically the host plant leading to its death [19]. Flowers offer the necessary moisture and a nutritious substrate for bacterial growth [20], and their colonization is a critical step in the spread of fire blight [21]. The bacterium may attain considerable population sizes, up to $10^{7} \mathrm{CFU}$ flower $^{-1}$, on hypanthia and pistil tissues, exploiting nectarthodes and other natural openings as the main entry points for the plant infection process [22-24]. Once floral tissues have been colonised, E. amylovora can be found also in anthers and pollen grains [25].

Honeybee-mediated dispersal of E. amylovora was demonstrated during the 1930s $[15,26]$. Flower-to-flower spread, rather than hive-to-flower contamination, is believed to play a significant ecological role, due to the limited survival time of the bacterium in hive conditions [27]. The pollinator-mediated pathogen spread efficiency depends on several aspects, including the relative attractiveness of healthy and infected plants. This factor may be affected by pathogen-induced changes in floral phenology and longevity, chemical composition of nectar and release of VOCs [8]. In addition, the relative proportion of pollinator visits on healthy versus diseased flowers and the pathogen population on the flower tissues and on visiting pollinators contribute to the probability of the insect to become a vector of the disease and to spread it to uninfected plants [28, 29].

Besides pollinator attraction, specific VOCs originate from the interaction of symbiotic or pathogenic microbes with the host plant, and may elicit plant growth and/or defence [30]. Airborne signals play a particular role in plant ecology, since they may spread independently of plant cell metabolism, sink/source balance and water flows [31], allow a long-ranged communication among neighbouring plants [32], attract symbiotic organisms [33], or exert a direct toxicity against plant-associated microbes [34, 35].

The system formed by apple (Malus $\times$ domestica Borkh.), its pathogen E. amylovora (Burrill) and the generalist pollinator A. mellifera provides a good bio/ecological model and, at the same time, has plenty of practical implications for horticultural practice. In fact, apple is among the most widely cultivated fruit trees in temperate areas (FAO data, 2016), and the process of domestication induced relatively little divergence in comparison to the wild ancestor species, M. sieversii [36, 37]. Finally, insectmediated pollination is required both in orchard conditions and in the wild [38].

Two alternative hypotheses were tested in the present study: (i) whether E. amylovora attracts honeybees to facilitate its own spread, or (ii) whether the coevolution of honeybees with flowering plants enabled mechanisms to prevent bacterial spread. The foraging preferences of honeybees on apple flowers inoculated with E. amylovora were determined by means of several, independent methods, also accounting for previous training of the bees on control (healthy) or inoculated flowers. Some of the nutritional and sensory determinants driving the honeybees' choice were investigated, with special regard for the characterization of apple flower scent. To this purpose, two sampling techniques and two GC-MS columns with complementary chemical specificity were used in this work, since the analytical settings (such as the GC-MS equipment and the chromatographic column) highly influence the qualitative and quantitative determination of VOCs [39, 40], and their insufficient exploratory power may prejudice the study of plant VOC profiles and ecological interactions based on them.

The results indicate that the spread of fire blight may be promoted by VOCs-mediated interaction between apple plants and pollinators.

\section{Materials and methods}

\section{Biological material}

Pollenizer apple plants (Malus $\times$ domestica Borkh.) about 1.5 $\mathrm{m}$ tall and grafted on M9 rootstocks, were purchased at Salvi Vivai (Ferrara, Italy) and maintained dormant at $4{ }^{\circ} \mathrm{C}$ in the dark until use. Before the experiment, the plants were placed in $20 \mathrm{~L}$ pots on a substrate obtained by mixing $1: 1(\mathrm{v} / \mathrm{v})$ peat and sand, with standard drip irrigation $\left(1.33 \mathrm{~L} \mathrm{day}^{-1}\right.$ plant $\left.^{-1}\right)$. No fertilization was applied for the duration of the experiment. The peat mineral concentration declared by the manufacturer was: $\mathrm{NH}_{4}{ }^{+} 25 \mathrm{~g} \mathrm{~m}^{-3}, \mathrm{NO}_{3}{ }^{-} 35 \mathrm{~g} \mathrm{~m}^{-3} ; \mathrm{P}_{2} \mathrm{O}_{5} 104 \mathrm{~g} \mathrm{~m}$ ${ }^{-3}, \mathrm{~K}_{2} \mathrm{O} 120 \mathrm{~g} \mathrm{~m}^{-3}$; $\mathrm{MgO} 12 \mathrm{~g} \mathrm{~m}^{-3}$; micronutrients $25 \mathrm{~g} \mathrm{~m}^{-3}$. Plants were acclimated in a greenhouse till bud break and subsequently transferred to field conditions just before blooming.

The bacteria employed in this experiment were Erwinia amylovora strain CFBP 1540 (holotype) and Pantoea agglomerans strain P10c, commercially used as a biological control agent [41]. The bacteria were grown in liquid LuriaBertani medium at $27^{\circ} \mathrm{C}$ under moderate shaking (100 rpm).

Honeybees (Apis mellifera ssp. carnica Pollmann) were kept in four standard 10-frames Dadant-Blatt beehives for nomad beekeeping. In each beehive, the brood was spread over five frames. Water was provided by a top-feeder and by placing pots filled with water nearby the hives.

\section{Field experimental setup}

The experiments took place between May and June in Bologna (Po river valley, Italy, 4432'48.62" $\mathrm{N}-11^{\circ}$ 24'58.43" E; 32 m.a.m.s.l). 
To prevent the uncontrolled diffusion of E. amylovora in the environment by contaminated bees or water leak, a tunnel was built with anti-aphid net (Antiafide 20/10 Mesh 50, Artes Politecnica s.r.l, Vicenza, Italy). The floor was covered with an impermeable plastic film. The tunnel dimensions were $18 \mathrm{~m} \times 3.50 \mathrm{~m} \times 5.50 \mathrm{~m}$, with an internal longitudinal septum dividing it in two halves. Each half contained 20 potted apple plants, irrigated daily with $1.5 \mathrm{~L}$ of water.

In one half of the tunnel, the 20 apple plants were inoculated with E. amylovora by spraying a $10^{7} \mathrm{CFU} \mathrm{mL}^{-1}$ bacterial suspension on the flowers. The 20 plants in the other half were mock-inoculated with water (control). Each plant was labelled with a randomly generated univocal code to allow a blind assessment of honeybees foraging preferences by operators. The flowers that were still closed at the time of inoculation were manually removed. After the inoculation, two beehives were introduced in each half of the tunnel, and bees were trained to forage either on control or inoculated flowers. Two nights later, the inoculated and non-inoculated control plants were distributed between the two halves of the tunnel, placing them alternately on two rows of 10 plants (Supplementary Information 1a).

\section{Assessment of honeybee foraging preferences}

During the preference tests, both inoculated and noninoculated control plants were presented in equal number and distance to bees trained on either plant group. Three independent methods were chosen in order to demonstrate honeybee preferences for control or inoculated flowers.

The first one consisted in the direct counting of the number of visits on each plant per time unit. All the plants were monitored at the same time for one hour, dividing the observations in 10-minute turns. During each turn, one operator surveyed 4 neighbouring plants randomly assigned. Counts were taken at $9 \mathrm{AM}$ and $4 \mathrm{PM}$ on the first day, and at 9 AM on the second day. The spread of $E$. amylovora on inoculated and non-inoculated flowers was monitored at each assessment of honeybee foraging preference. Sixty flowers were sampled from each plant and externally washed in sterile $10 \mathrm{mM} \mathrm{MgSO}$. The wash was plated on agarized Miller \& Schroth medium [42] amended with cycloheximide $\left(1 \mathrm{mg} \mathrm{L}^{-1}\right)$. Endophytic E. amylovora population was assessed after surface-sterilization of flowers, by successive $1 \mathrm{~min}$ sonication washes in $70 \%$ ethanol, $0.1 \% \mathrm{NaOCl}$, and twice in autoclaved water. Subsequently, samples were homogenised in $10 \mathrm{mM} \mathrm{MgSO}_{4}$ and the bacterial population was assessed as previously described. In addition, E. amylovora population was quantified by qPCR with a StepOne Plus Real-Time PCR instrument (Applied Biosystems, Foster City, CA, USA), using primers designed on the plasmid pEA29 [43] and a SYBR Green- based chemistry. Pure E. amylovora cultures from $10^{2}$ to $10^{8} \mathrm{CFU} \mathrm{mL} \mathrm{mL}^{-1}$ were included as standards.

A second approach to evaluate honeybee preference was based on the use of the marker bacterium, P. agglomerans strain P10c, which was carried by honeybees on the visited flowers during their foraging activity. Competition effects by E. amylovora on this strain were previously verified to be negligible. $P$. agglomerans $\mathrm{P} 10 \mathrm{c}$ was mixed as a lyophilised powder with pollen and pulverized coal to a titre of $10^{7} \mathrm{CFU} \mathrm{mg}{ }^{-1}$, and dispensed through a pollen insert placed on the hive, as described by Vanneste [44] in order to dust each honeybee exiting the hive. Two hours later, 15-20 honeybees exiting the hive and 30-100 flowers were singularly sampled. On these samples, $P$. agglomerans $\mathrm{P} 10 \mathrm{c}$ populations were determined by extracting the bacteria in $10 \mathrm{mM} \mathrm{MgSO}_{4}$, producing 1:10 sequential dilutions of the extract, and plating 3 drops of $10 \mu \mathrm{L}$ for each dilution on agarized LB medium amended with cycloheximide, rifampicin and streptomycin $\left(1 \mathrm{mg} \mathrm{L}^{-1}\right)$ to discriminate P10c from other $P$. agglomerans strains. In addition, a qPCR protocol was adopted for the same purpose. DNA was extracted from flowers and processed as previously described [45]. As a quantification standard, pure $P$. agglomerans cultures from $10^{2}$ to $10^{8} \mathrm{CFU} \mathrm{mL}^{-1}$ were included in each qPCR assay performed. Data were normalized for P. agglomerans populations naturally occurring in field conditions. A preliminary experiment, performed in the same tunnel to estimate the efficiency of marker bacterium dissemination by honeybees, resulted in approximately $11 \%$ contaminated flowers per hour when the bacterium was dispensed through the pollen insert. All the honeybees collected during the experiment were contaminated by $P$. agglomerans strain $\mathrm{P} 10 \mathrm{c}$, with a population of $10^{(4.2 \pm 1.3)} \mathrm{CFU}$ insect $^{-1}$.

The third method consisted in the determination of $E$. amylovora population sizes in the pollen loads removed from the pollen baskets of the foraging bees returning to the hive. E. amylovora population was assumed to be proportional to the number of visits on infected flowers. Negative and positive controls were respectively represented by pollen collected during the training by bees exposed only to control or inoculated flowers. E. amylovora populations were determined both by enumeration on agarized medium and by qPCR, as previously described.

To determine whether VOCs were the major driver for to the honeybee foraging preferences in our system, a blind choice test was performed. For this purpose, flowering branches $(6$ inoculated +6 control, alternately positioned in a row) were enclosed in white cylinders made of perforated paperboard, to allow gas exchanges while hiding visual cues. A Petri dish containing feeding solution $(70 \% \mathrm{w} / \mathrm{v}$ sucrose and $0.3 \% \mathrm{w} / \mathrm{v}$ citric acid in water) was placed on top of each cylinder, and the number of visits per time frame 
(10 minutes) was recorded. The experiment was replicated three times for two subsequent days, starting three days after inoculation.

\section{Flower morphology and radiance}

Colour characteristics, irradiance under UV light and size were measured on 9 mature control and inoculated flowers. For this purpose, recently opened flowers were labelled before inoculation to assure age uniformity between control and inoculated samples. The flowers were collected on the last day of the experiment, and their biometric characteristics were evaluated with a Nikon SMZ25 fluorescence microscope (Nikon Instruments Corporation, Tokyo, Japan).

\section{Nectar composition}

To evaluate whether pathogen inoculation affects the nutritional reward of flower nectar, sugar and organic acid composition of the floral exudates were determined by gaschromatography coupled to a flame ionization detector. Twenty flowers, taken from different plants (10 inoculated and 10 control) in the tunnel, were singularly sampled and processed as described by Bartolozzi et al. [46]. Briefly, the sample was washed in an extraction buffer containing 10 $\mathrm{mM}$ imidazole in an ethanol:water:acetone $(50: 40: 10$ by volume) mixture for one hour. Subsequently, $500 \mu \mathrm{L}$ of the extract were added to the same volume of internal standard (phenyl- $\beta$-glucopyranoside, $2 \mathrm{gL}^{-1}$ in the same ethanol: water:acetone mixture) in a chromatography vial. The sample was dried under air stream, and derivatized with the successive addition of $400 \mu \mathrm{L}$ pyridine, $200 \mu \mathrm{L}$ hexamethyldisilazane and $100 \mu \mathrm{L}$ trimethylchlorosilane. After incubation at $60{ }^{\circ} \mathrm{C}$ for $2 \mathrm{~h}$, the samples were analysed with a Varian 3900 equipment, mounting a CP8401 auto-injector (Varian Inc., Palo Alto, USA) and a 30-m long CP-Sil 5 CB column. Helium was used as the carrier gas. The flame ionization detector used hydrogen, nitrogen and oxygen. Injector and detector temperatures were 125 and $300{ }^{\circ} \mathrm{C}$, respectively. For each run, with a duration of $17.75 \mathrm{~min}, 1$ $\mu \mathrm{L}$ of sample was injected.

\section{Pollen production and quality}

Since pollen may be a food source for honeybees, production and viability of pollen were tested after inoculation with E. amylovora. Greenhouse-grown flowering plants were spray-inoculated with a $10^{6} \mathrm{CFU} \mathrm{mL}^{-1}$ E. amylovora suspension, as described for field experiments. Mockinoculated plants were used as controls. Three days after inoculation, one hundred flowers from inoculated or control plants were collected. Anthers were excised in sterile conditions, and dried at $35{ }^{\circ} \mathrm{C}$ for $24 \mathrm{~h}$. Subsequently, the pollen was separated by means of a $55-\mu \mathrm{m}$ mesh sieve. The pollen was weighed, and the size and morphology of pollen grains were evaluated with a Nikon SMZ25 fluorescence microscope at $200 \times$ magnification.

Pollen germinability was quantified by dropping pollen grains on sterile agarized sucrose $\left(100 \mathrm{~g} \mathrm{~L}^{-1}\right)$ solution. After $5 \mathrm{~h}$ at $25^{\circ} \mathrm{C}$ under non-direct daylight, the percentage of germinated pollen grains was evaluated by microscopical observation.

\section{VOCs emissions from infected flowers}

Exploratory analyses were performed on VOC release from flowers of plants maintained in tunnel. VOC samplings were performed between 10 and 11 AM. Each sample was formed by one flower cluster per plant, included in an odourless polyethylene bag $\left(\mathrm{Cuki}^{\circledR}\right.$, Cofresco, Volpiano, Italy); VOC emissions were adsorbed on solid phase microextraction (SPME) fibres (DVB/CAR/PDMS, $2 \mathrm{~cm}$, 23-Gauge, Supelco, Bellefonte, USA) exposed in the bag for one hour. Subsequently, VOC analysis was carried out on two GC-MS instruments mounting columns with different polarity. The first was a 7890A-5975C (Agilent Technologies, Santa Clara, USA) equipped with a HP-5MS, non-polar column $(30 \mathrm{~m} \times 0.25 \mathrm{~mm} \times 0.25 \mu \mathrm{m}$ I.D $)$. The oven starting temperature $\left(50^{\circ} \mathrm{C}\right)$ was held for $1.5 \mathrm{~min}$, followed by an increase of $7.5^{\circ} \mathrm{C} \mathrm{min}{ }^{-1}$ until a temperature of $250^{\circ} \mathrm{C}$ was reached and held for $10 \mathrm{~min}$. The carrier gas was helium at a flow rate of $1.2 \mathrm{~mL} \mathrm{~min}^{-1}$ and a speed of $39.92 \mathrm{~cm} \mathrm{~s}^{-1}$. The second instrument was a Shimadzu GCMS-QP2010 Plus (Shimadzu, Tokyo, Japan) mounting a RTX-WAX fused-silica (polar) capillary column $(30 \mathrm{~m} \times$ $0.25 \mathrm{~mm}$ i.d. $\times 0.25 \mu \mathrm{m}$ ) coated with polyethylene glycol (PEG) (Restek, Bellefonte, PA, USA). The oven heating program was: $45^{\circ} \mathrm{C}$ for $10 \mathrm{~min} ; 4^{\circ} \mathrm{C} \mathrm{min}^{-1}$ temperature increase to $200{ }^{\circ} \mathrm{C} ; 200{ }^{\circ} \mathrm{C}$ for $8 \mathrm{~min}$, under a helium flow of $1.0 \mathrm{~mL} \mathrm{~min}^{-1}$. Identification of volatile compounds was performed according to their retention indices and mass spectra by comparison with the NIST/EPA/NIH Mass Spectral Database (NIST 11, National Institute of Standards and Technology, Gaithersburg, USA). A commercially available mixture of n-alkane standards (nC9 - nC20, Sigma-Aldrich) was used to calculate the linear retention indices (LRI) of the identified VOCs [47]. The obtained LRI values were compared with the reference LRI values present in the NIST Chemistry WebBook (NIST Chemistry WebBook, 2005) and corresponding to the same columns used in our experiments. In case of multiple literature LRI values for the same compound, the median values were used instead. When compounds had asymmetrically substituted carbons, the isomeric compositions were not determined (e.g. limonene, linalool). Five samples for 
inoculated and for control flowers were analysed with each GC-MS equipment.

Since the first GC-MS equipment was more suitable for the detection of compounds present in the VOC blend, this instrumental set was used to analyse also the VOCs samples collected by closed-loop stripping analysis (CLSA), which allows a better quantification of the adsorbed compounds. Five samples were taken for inoculated and control flowers. A flower cluster from each plant was enclosed within an odourless polyethylene bag. Air samples were collected using an adsorbent trap (glass tube, $6.5 \times 0.55 \times 0.26 \mathrm{~cm}$, loaded with $1.5 \mathrm{mg}$ activated charcoal; CLSA filter LR-type; Brechbühler AG, Schlieren, Switzerland). The trap was fitted to a $12 \mathrm{~V}$ graphite vacuum pump (Fürgut, Tannheim, Germany) using a short Teflon tube. The pump circulated air at a rate of ca. $1 \mathrm{~L} \mathrm{~min}^{-1}$ within the VOC-bag. Samples were collected daily from $10 \mathrm{AM}$ to $1 \mathrm{PM}$. The collected VOC samples were eluted from the adsorbent traps with $100 \mu \mathrm{L}$ GC grade dichloromethane (Sigma-Aldrich, Milan, Italy) and stored at $-80^{\circ} \mathrm{C}$ prior to GC-MS analysis.

\section{Statistical analysis}

Statistical analyses were performed using Statistica (ver. 5.0, Statsoft Inc., 1995, Tulsa, USA) and R 3.3.3 (R Foundation). Honeybee preference data were expressed as the percentage of visits between inoculated and control plants; significance of differences was calculated by the binomial distribution test. Student's $T$-test was used for differences in nectar composition. $\chi^{2}$-test was employed to assess the significance of differences in P10c incidence and E. amylovora infection rates. GC-MS data were analysed with Levene and Student's $T$-tests. Principal Component Analysis (PCA) of variance and Partial Least Square-Discriminant Analysis (PLS-DA) were performed on these data.

\section{Results}

\section{Honeybee foraging preferences}

To determine whether the inoculation with E. amylovora could affect flower attractiveness, honeybees were included in a net tunnel with inoculated or non-inoculated (control) plants. Their foraging preference was evaluated by three independent methods, i.e. counting honeybee visits on inoculated or control flowers, experimentally loading honeybees with a marker bacterium ( $P$. agglomerans strain P10c) and measuring the incidence of the marker on flowers, and quantifying E. amylovora population in the harvested pollen. By all the methods tested, a preference of honeybees for control flowers emerged.

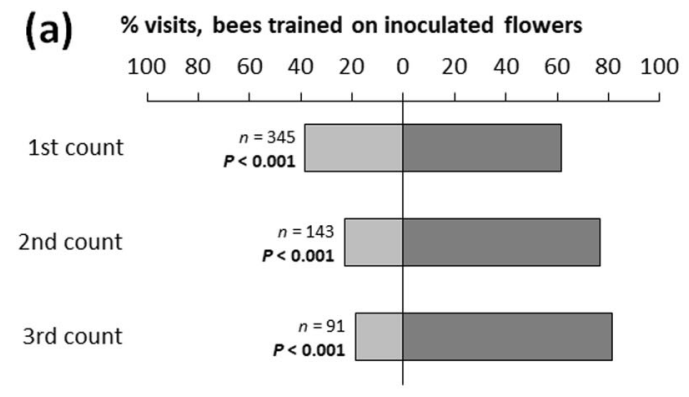

(b) \% visits, bees trained on control flowers

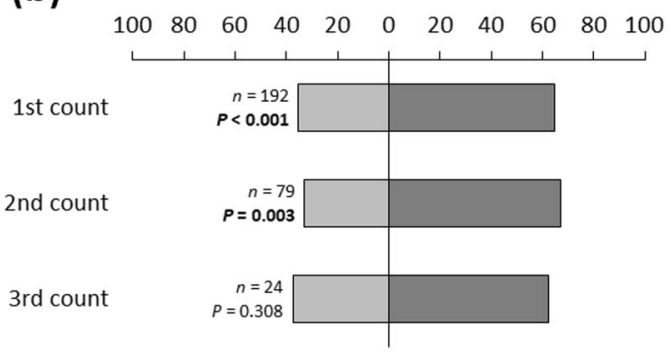

inoculated flowers

Fig. 1 Assessment of visit preference of forager honeybees, Apis mellifera $\mathrm{L}$., after training on inoculated $(\mathbf{a}, \mathbf{c})$ or control $(\mathbf{b}, \mathbf{d})$ flowers. Preferences were measured by the proportion of direct counts of visits on inoculated or control plants $(\mathbf{a}, \mathbf{b})$, or by isolation of a marker bacterium, P. agglomerans strain P10c (c, d), experimentally loaded
(C) incidence of marker bacterium, bees trained on inoculated flowers

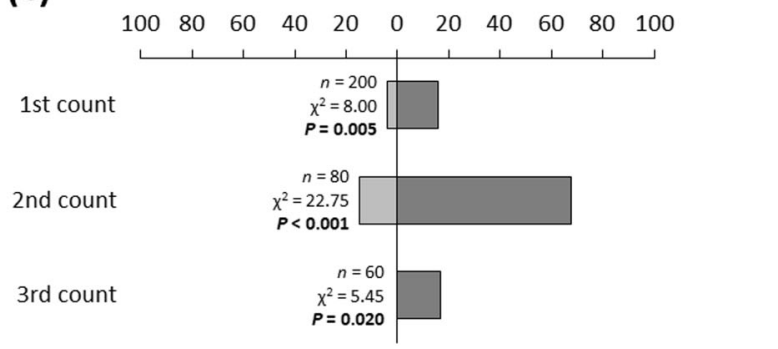

(d) incidence of marker bacterium, bees trained on control flowers

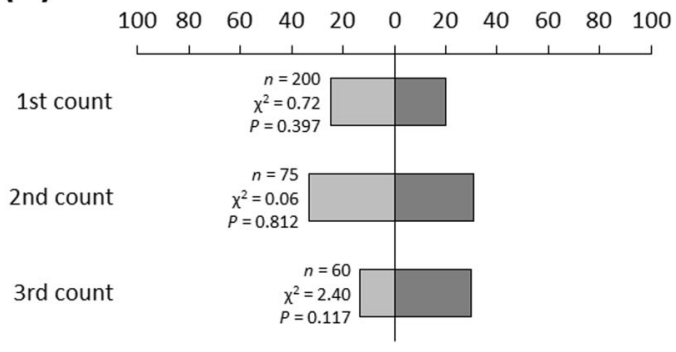

control flowers

onto honeybees and delivered on visited flowers. Significantly different $(P<0.05$, indicated in bold) preferences were determined according to the two-tailed binomial distribution test $(\mathbf{a}, \mathbf{b})$ or to the $\chi^{2}$ test $(\mathbf{c}, \mathbf{d})$ 
The proportion of visits on control flowers was above $60 \%$ in 5 out of 6 direct counts (Fig. 1a, b), and reached $82 \%$ for bees previously trained on inoculated flowers. Similar data were obtained by assessing the presence of the marker bacterium P. agglomerans strain P10c (Fig. 1c, d) on flower samples, resulting from honeybee-mediated spread. In fact, the incidence of the marker bacterium was significantly higher on control flowers when honeybees trained on inoculated flowers were used, while this preference could only be noticed on the third count when the bees were trained on control plants. Quantification of P10c by qPCR confirmed this result. The third method used to evaluate honeybee preferences consisted in the quantification of E. amylovora population in the harvested pollen. $E$. amylovora was not detectable in any of the pollen samples collected from the hives, except the positive control (Table 1).

During the experiments, the incidence of E. amylovora constantly increased on non-inoculated control flowers (Fig. 2). The ratio of secondary infection was determined as the percentage of non-inoculated flowers harbouring an endophytic E. amylovora population. At the end of the trials, $10 \%$ and $27 \%$ of non-inoculated flowers had become infected after the foraging activity of honeybees trained on inoculated and non-inoculated plants, respectively. All the sampled inoculated flowers were contaminated, with an average population of $10^{(6.8 \pm 1.0)}$ CFU per flower. Approximately $80 \%$ of them hosted an endophytic E. amylovora population during the whole experiment.

\section{Sensorial and nutritional determinants of attraction}

Having observed a preference of honeybee for noninoculated flowers, we investigated the relative importance of sensorial cues and nutritional rewards in driving their foraging behaviour.

At the time of the experiments, visible symptoms of fire blight had not appeared yet either on flowers or on other tissues. Apple flowers were characterized by five completely white petals (Supplementary Information 1b). Generally, green sepals were visible among petals. Microscope observation under visible/UV light of whole flowers confirmed the similarity in shape, size and colour between control or inoculated flowers (Supplementary Information 1c). Blind choice test, performed with covered flowering branches, confirmed that non-trained honeybees were more attracted to control (with over $60 \%$ of preference) than to inoculated flowers even in absence of visual cues (Fig. 3).

Table 1 Bacterial population of Erwinia amylovora in the pollen collected by forager bees in each day of observation, according to their training on control or inoculated flowers

\begin{tabular}{llll}
\hline Collection time & Honeybee training & Pollen collected $\left(\mathrm{mg} \mathrm{hour}^{-1}\right)$ & E. amylovora $(\mathrm{CFU} \mathrm{mg}$ \\
\hline Training (pre-experiment) & inoculated flowers & 2.7 & $6.5 \times 10^{7}$ \\
& control flowers & 93 & 0 \\
Day 1 & inoculated flowers & 4.0 & 0 \\
& control flowers & 3.5 & 0 \\
Day 2 & inoculated flowers & 5.1 & 0 \\
& control flowers & 0.0 & 0 \\
\hline
\end{tabular}

(a)

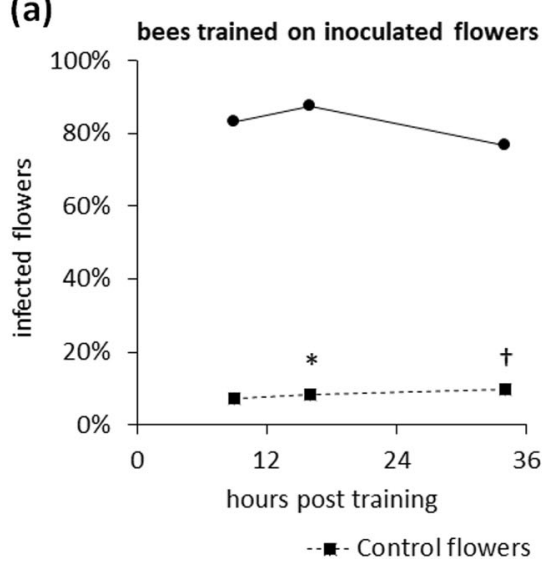

Fig. 2 Disease incidence in experimentally inoculated (solid line) and in control (dashed line) flowers exposed to bee-mediated vectoring of the pathogen. The disease incidence was calculated as the percentage of flower harbouring a detectable endophytic E. amylovora population.

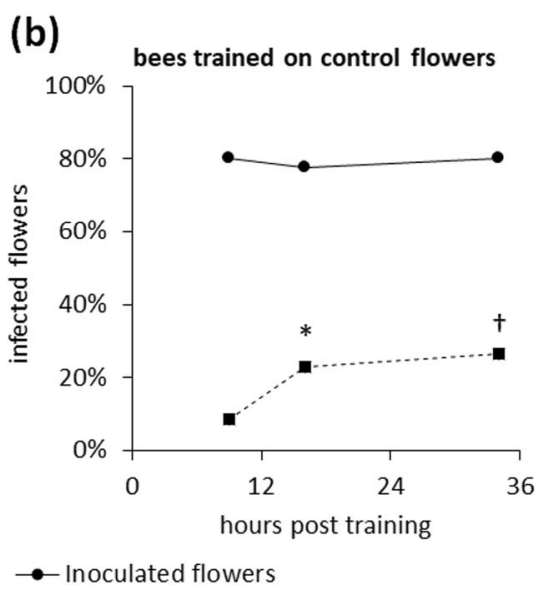

Experiments were performed with bees trained either on inoculated (a) or control (b) plants. Data pairs marked with the same symbol in the two panels are significantly $(P<0.05)$ different according to $\chi^{2}$-test for $n=60\left(*-\chi^{2}=7.38 ; \dagger-\chi^{2}=8.25\right)$ 


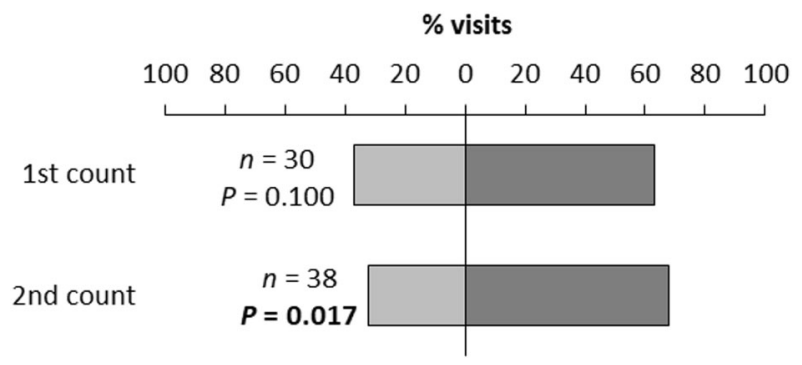

inoculated flowers

control flowers

Fig. 3 Assessment of visit preference of naive forager honeybees, Apis mellifera L., by the proportion of direct counts of visits on inoculated or control branches. The branches were covered by a white netting, preventing visual flower detection but not odour perception. Significantly different $(P<0.05$, indicated in bold) preferences were determined according to the two-tailed binomial distribution test

No significant differences emerged between sugar contents in control and inoculated flowers. Only malic acid showed a slight (approx. 20\%) reduction in infected samples (Supplementary Information 1d). Both control and inoculated flowers produced similar amounts of pollen $\left(49.8 \pm 6.8\right.$ and $50.4 \pm 8.4 \mathrm{mg} \mathrm{g}^{-1}$ of fresh anther tissue, respectively).

\section{VOC composition of flower blends}

Since visual/UV cues or nutritional rewards could not allow the discrimination of inoculated from control flowers, nor explain honeybee preference, flower VOC emissions were characterised by GC-MS.

In a first step, SPME sampling allowed to identify 93 compounds. The differences between control and inoculated flowers were mainly quantitative, since no compounds were exclusive to either inoculated or control flowers (Fig. 4 and Supplementary Information 2). 1-penten-3-ol and 3-(E)hexen-1-ol showed a significantly higher emission from inoculated flowers.

To substantiate these findings, GC-MS analysis was repeated after semi-quantitative CLSA sampling (Fig. 4 and Supplementary Information 2), and the results were analysed by PLS-DA. The overall VOC profiles showed an inoculation-dependent distribution on the first principal component of variance (Fig. 5). The inoculated samples were characterised by several sesquiterpenes (farnesene isomers, curcumene, copaene) and methyl salicylate, while control flowers were characterised by a slight prevalence of phenolic compounds (such as benzaldehyde, acetonitrile and benzyl alcohol), aldehydes (nonanal, decanal) and (Z)jasmone (Fig. 5b).

\section{Discussion}

\section{Influence of inoculation on honeybee foraging behaviour}

In addition to long-known functions in the interaction between plants and insects [6], the involvement of VOCs in plant communication with symbionts and defence against pathogens has emerged [30, 31, 48]. Many pollinatorvectored pathogens have been described previously [8], but only few of them are bacterial pathogens, mainly belonging to the genus Erwinia. In addition, the influence of VOC changes induced by pathogen infection on pollinators' behaviour was investigated only for two highly specialised fungal pathogens [10-13].

Using three independent experimental procedures, we tested whether flower infection by E. amylovora would increase honeybee visit rates on apple flowers. In contrast to our experimental hypothesis, a preference of honeybees for control over inoculated flowers emerged, since visits on control plants were more frequent and non-inoculated flowers presented a higher incidence of the marker bacterium ( $P$. agglomerans $\mathrm{P} 10 \mathrm{c}$ ), delivered by the insects. Although pollen production was not affected by the pathogen, E. amylovora was detected in the collected pollen only during training (Table 1), further confirming that pollen was almost exclusively harvested from control flowers.

Training the bees over infected plants further increased their preference for control flowers. In fact, bees trained on inoculated flowers increasingly shifted to control flowers, while bees trained on control flowers visited inoculated ones with a lower, but steady frequency. This observation suggests that a learning process may contribute to the avoidance of infected flowers.

During the experiment, as a result of secondary bacterial spread, a significant share of the non-inoculated flowers became endophytically infected with E. amylovora (Fig. 2). This may have caused, in principle, a reduced discrimination by foraging bees between experimentally inoculated and secondarily infected control flowers. However, such reduction did not occur, while a steadily increasing preference for non-inoculated blossoms was observed. This observation suggests that repulsive effects would grow in intensity with the progression of bacterial infection, i.e. recently infected flowers, harbouring a lower pathogen load, would be less repulsive than those at later infection stages.

\section{Determinants of honeybee foraging preferences}

In this work, visual cues and nutritional rewards did not differ significantly between control and inoculated flowers, 


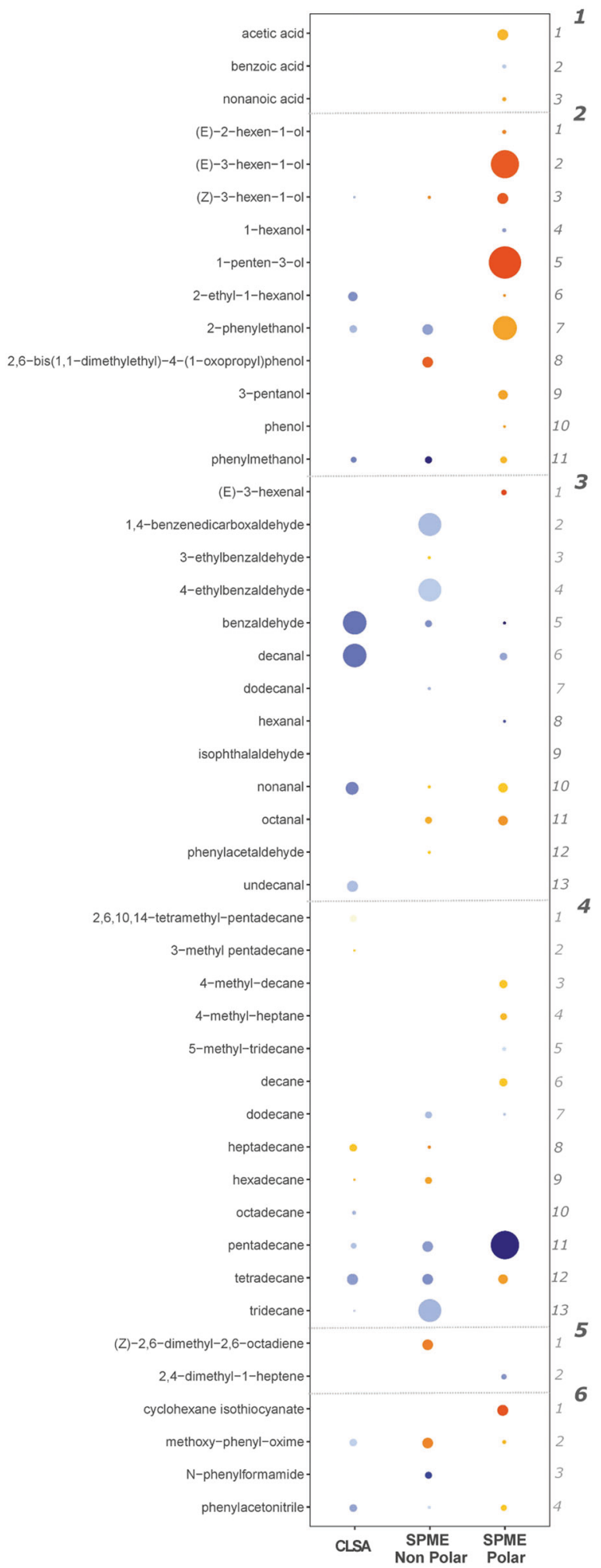

Fig. 4 List of volatile compounds identified by GC-MS analysis in the three experimental conditions adopted in this work (CLSA sampling plus separation on non-polar column, SPME sampling plus separation on nonpolar column, SPME sampling plus separation on polar column). The chroma scale indicates the relative variation of emission from inoculated flowers, in comparison to control ones (expressed as $\log _{2}$ of peak area

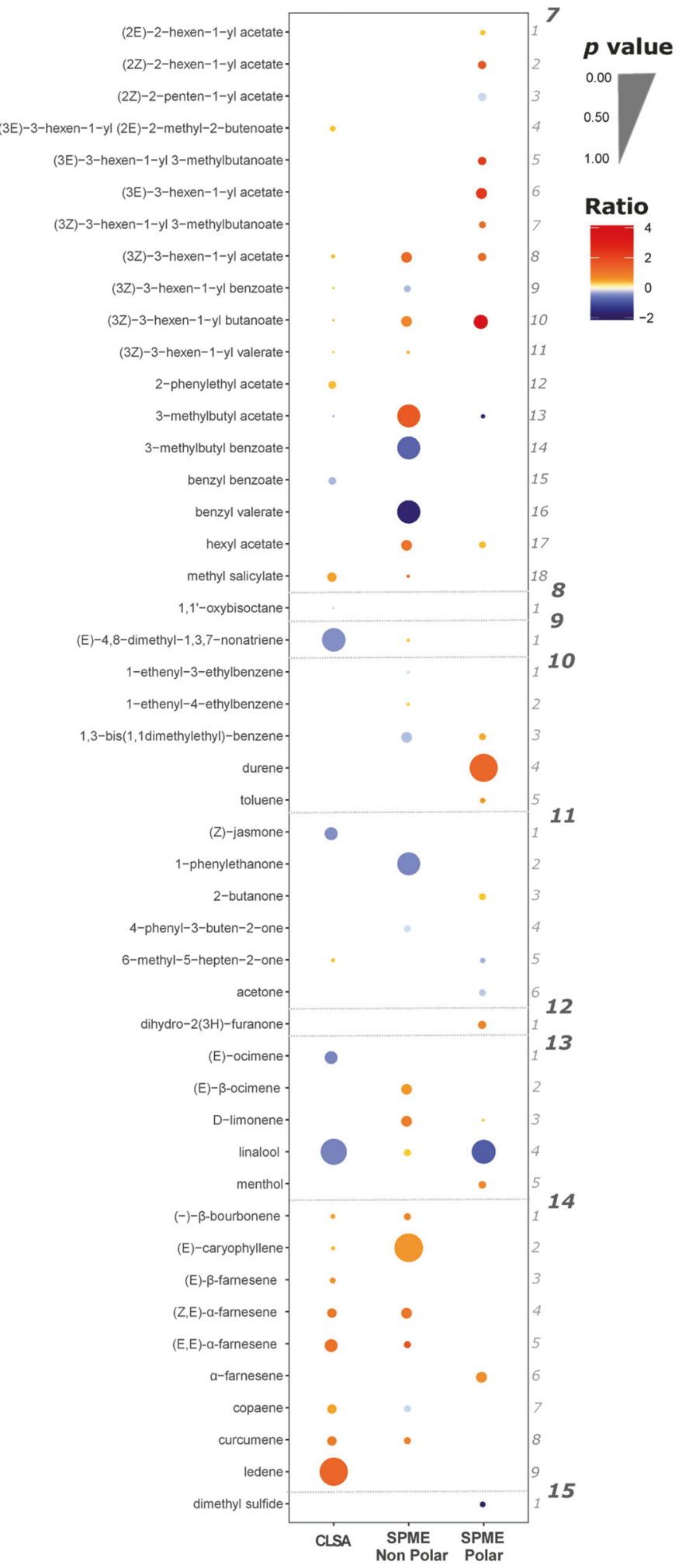

ratio). The $P$-value of differences between control and inoculated flowers is proportional to the relative circle diameter. The compounds are divided in the following chemical groups: 1 - acids; 2 - alcohols; 3 - aldehydes; 4 - alkanes; 5 - alkenes; 6 - nitrogen compounds; 7 - esters; 8 - ethers; 9 homoterpenes; 10 - benzene compounds; 11 - ketones; 12 - furans; 13 monoterpenes; 14 - sesquiterpenes; 15 - sulfur compounds 


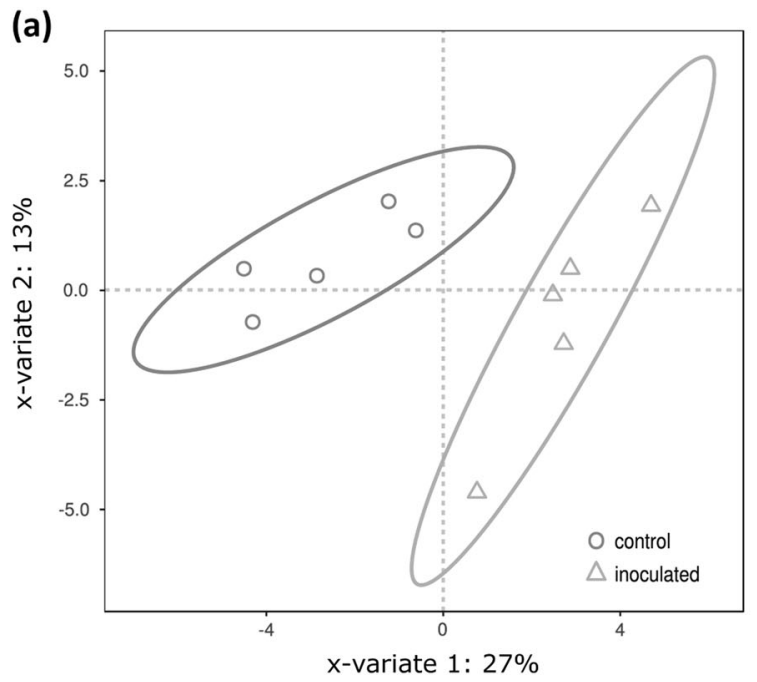

Fig. 5 Two-dimensional partial least square-discriminant analysis (a) and relative loadings (b) obtained from GC-MS analyses of volatile compounds emitted by control or inoculated flower samples analysed

leaving the difference in honeybee visit rates unexplained. Instead, differences in VOC profiles were observed, suggesting that honeybees can discriminate the flowers basing on olfactory signals.

In contrast to previous studies [49-51], in which a single experimental equipment was used, this work employed both a polar and a non-polar GC-MS column. This set-up allowed a comprehensive characterization of apple flower scent, including over 90 different compounds. Even though both of our analytical settings were broad-range and exploratory, very little overlapping was obtained in their outcomes, evidencing that a description of complex blends should rely on multiple and complementary methods.

Due to the potential biological importance of the compounds identified with the non-polar column (including a variety of terpenoids and phenolic compounds), gas sampling with CLSA sorbent fibres was applied to this GC-MS setting for the semi-quantitative determination of the VOCs. A high variability in flower VOC emissions was observed, possibly due to differences in flowerage and/or stage of infection, predominating over those induced by the infection status. Although closed and senescent flowers had been removed before inoculation, slight differences in flower age, or a senescence rate promoted by infection may have resulted in an asynchronous phenology. In addition, on one hand, inoculation led to successful infection only in part (about $80 \%$ ) of the flowers, on the other hand, the bacterium spread to control flowers during the experiment (Fig. 2).

Such reasons may explain why differences in the emission of single compounds were generally poorly significant, with a modest variation (from 75 to 200\%) in emission rates from inoculated flowers compared to controls. However, the analysis of the overall VOC profiles by PLS-DA (Fig. 5)

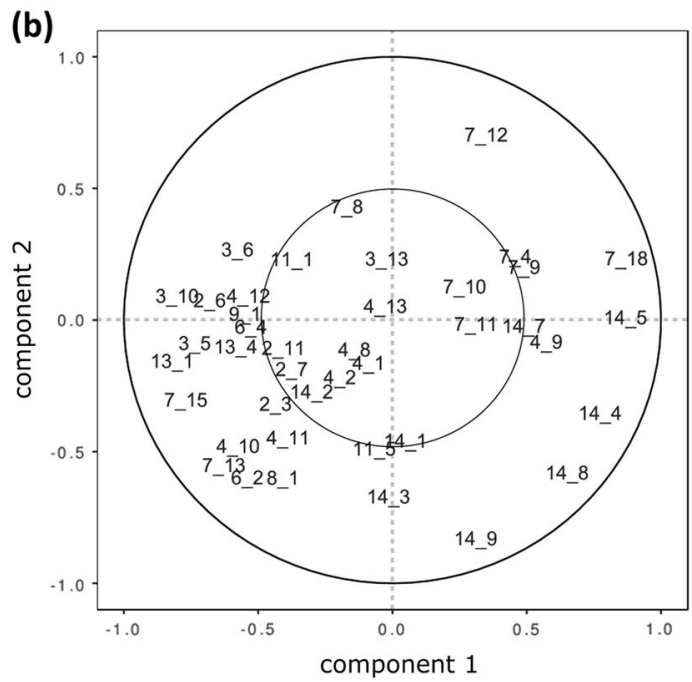

by CLSA-GC-MS with non-polar column. Code numbers in (b) indicate the corresponding compounds presented in Fig. 4

allowed to discriminate control from inoculated flowers, indicating that, even in the absence of single characterising compounds, inoculated flowers could be recognised by their global VOC emissions. Methyl salicylate and several terpenoids ( $\alpha$-farnesene isomers, ledene, curcumene), which are considerably ( 257 to $480 \%$ ) more emitted by inoculated flowers, contributed most to the separation of the two classes.

\section{Plant-pollinator relations mediated by VOCs}

Some compounds, although normally emitted also by healthy and vegetative tissues [52], were differentially released from control and inoculated flowers, suggesting that odour signals may drive honeybees foraging preferences. According to previous studies [53-55], honeybees respond to a small subset of total floral VOCs, several of which were observed in this work: benzaldehyde, $(E)-\beta$ ocimene, linalool, nonanal, methyl salicylate, $(E, E)$ - $\alpha$-farnesene, phenylethyl alcohol and 2-phenylethyl acetate. In this study, $(E, E)-\alpha$-farnesene was one of the most strongly emitted compounds, and showed a dramatic surge after infection; other sesquiterpenes (copaene, (-)- $\beta$-bourbonene, $(E)$-caryophyllene, ledene, curcumene and farnesene isomers) followed the same trend. (Z)-3-hexen-1-yl acetate also showed major increases, but its effects on honeybee pollination were described as minor [56]. However, the higher intensity of VOC release by inoculated flowers did not positively correlate with honeybee harvesting preference. These conflicting observations could be explained either by a short-term associative learning or by a different response to a complex VOC mixture in comparison to single compounds. 
In the first case, preferences to specific VOC blends would be associated to differently rewarding flowers with a learning process. Generalist pollinators, such as bees, tend to use honest floral signals (i.e., those correlated to reward) as short-term associative cues [57]. Although only minor differences in nutritional rewards were found in this work, they may reflect a significantly reduced nutritional reward from infected flowers when taken together. Besides, the lower variability of VOC profiles from control flowers (Supplementary Information 1e) would promote associative learning and recognition of the target by honeybees, resulting in increased pollination specificity [58-60].

Alternatively, the intrinsic attractiveness/repulsiveness of the single components of the flower VOCs blend may additively concur to the overall pollinator preference. Previous studies [61] observed an attractive effect for linalool (more emitted by control flowers) and a repulsive effect of methyl salicylate (promoted by inoculation), in agreement with the data presented here. However, other compounds $((E)$-ocimene, $(Z)$-hexenyl acetate, caryophyllene, 1-penten3-ol, 1-hexanol) are known to elicit an electroantennographic signal, but their influence on honeybee attraction is neutral or undetermined [61, 62].

The relative concentration of the compounds, besides or instead of their chemical nature, may also determine whether the blend has, as a whole, an attractive or repulsive effect. In fact, perceptual properties of VOCs in a blend depend on the nature, concentration, variability and number of its components [59]. Overall, multiple decision mechanisms may coexist in honeybees.

\section{Flower responses to infection}

Some of the observed changes in VOC emissions, instead of affecting the plant-pollinator relations, may have a direct role in the plant-pathogen interactions. Among the compounds more emitted by inoculated flowers, $(E)$-caryophyllene was previously shown to express appreciable antimicrobial functions [35]. Similarly, the role of methyl salicylate in plant defences is well established [63]. Consistently with the antagonistic roles of jasmonates and salicylates, the abundance of $(Z)$-jasmone was generally lower in inoculated flowers. (Z)-3-hexen-1-ol and (Z)-3-hexen-1yl acetate are signals released after cell damage, eliciting defensive responses in distal tissues and neighbouring plants $[64,65]$. The induction of defensive responses mediated by specific VOCs resulting from microbe metabolism or plant cell damage [30, 32, 64] has remarkable consequences, allowing to spread the alert signal systemically and extending the signalling network to other neighbouring plants.

VOCs take part in the integration of signals coming from pathogens, microbial symbionts, pests and beneficial insects, to shape the plant's most appropriate adaptive response [48]. In our experiment, synergistic protective effects on apple plants may be attributed to the increased emission of certain compounds (most notably, methyl salicylate) after flower infection (Fig. 6). Firstly, they evoke plant defences more rapidly and diffusely than soluble signals transported through plant vessels, thus counteracting bacterial colonization [31, 63]. Secondly, their effect extends to neighbouring plants, lowering the chance of a successful secondary infection [32]. Altogether, VOCs released from infected flowers can be perceived by honeybees, driving their foraging preference to healthy flowers. However, such preference does not result in a complete avoidance of infected flowers. Occasional visits on infected flowers, followed by visits on healthy ones, may promote bacterial spread. Some of the changes in VOC emissions induced after inoculation with E. amylovora can be explained by a direct defensive function, but may not affect pollination (Fig. 6).

\section{Ecological importance of honeybee-mediated spread of $E$. amylovora}

In addition to their roles in plant defences, VOCs from infected flowers may deter honeybee visits, thus limiting pathogen transmission from host to host. This view is in agreement with a coevolution of apple plants with pollinators, enhancing the stability of the symbiotic system in

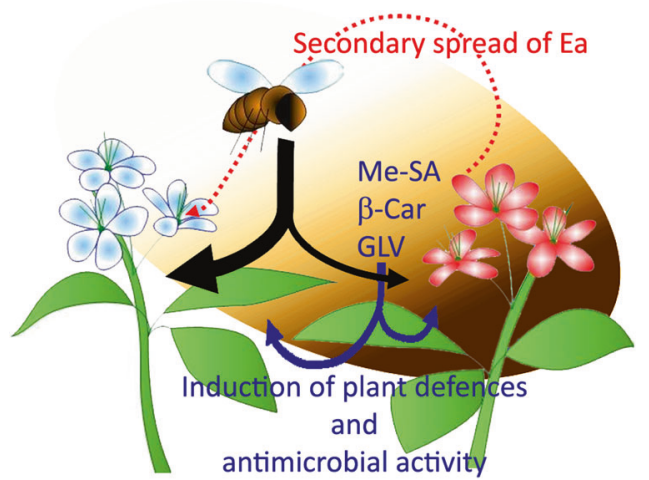

Fig. 6 Summary of the trilateral interaction occurring between host apple plants, the bacterial pathogen Erwinia amylovora and honeybees. Infected plants (shaded in red) present an alteration in their emission of volatile compounds. Some of these, such as methyl salicylate (Me-SA) and green leaf volatiles (GLV) are known to elicit plant defences both in the infected plant and in the surrounding ones $[64,65]$. Others, such as terpenes and $\beta$-caryophyllene ( $\beta$-Car), may exert a direct antimicrobial effect [35]. However, the same set of volatile compounds reduces the pollinator visit rate on infected flowers (black arrows, the size of the arrows is proportional to honeybee visiting preferences). As the deterrence effect is not complete, occasional honeybee visits on infected flowers may allow the pathogen to be vectored to the more frequently visited healthy flowers (red dashed arrow), contributing to its spread 
presence of perturbing agents such as the pathogen $E$. amylovora. However, different selective pressures are acting in this system. As generalist pollinators, honeybees may exploit a wide variety of flower species, basing their foraging activity on active exploration and learning rather than plant specificity $[53,57]$. In contrast, specialised pathogens compensate their narrow host range with a high efficiency of colonisation and infection [66]. As a result, a full protection of the system, resulting from the complete avoidance of infected flowers, is not achieved.

In this scenario, the pathogen may take advantage from the existing symbiosis between apple plants and honeybees, its modulation by defence-related compounds, and the learning capabilities of the insects to achieve a more efficient bacterial spread. Field observations on bacterial spread $[67,68]$ and E. amylovora aggressiveness [69] indicate that pollinating insects may take up from infected flowers, and subsequently deliver to healthy flowers, bacterial populations compatible with E. amylovora infection.

As an unexpected consequence, a reduced visit rate on infected flowers may result in a more efficient bacterial spread [28, 29]. In fact, once contaminated, an insect has a higher probability to visit several healthy flowers, delivering the pathogen to each of them. In addition, the honeybee learning process may further contribute to bacterial spread, since a higher share of insects may become contaminated before developing a preference for healthy flowers (Supplementary Information 3).

\section{Conclusions}

This work provides evidence that E. amylovora-infected flowers exert a lower attraction to honeybees, and VOC cues may be responsible for their discrimination from healthy flowers. Repulsive effects of some VOCs (e.g. methyl salicylate) released by inoculated flowers seemingly prevail over other attractive compounds. However, some aspects still need clarification concerning the biochemical details and the ecological consequences of honeybee pollination preferences.

The emission of deterring compounds by infected flowers, reducing the pollinator visit rate on them, may contribute to a wider spread of the pathogen, as honeybees increase their visits on healthy flowers after becoming contaminated. This ecological mechanism may be regarded as an effective strategy adopted by E. amylovora to turn plant defences to its own advantage.

Due to the limited survival of the bacterium in beehives and honeybee foraging behaviour, a honeybee-mediated, long-range or long-term E. amylovora spread, according to our observations, appears unlikely. In contrast, the incomplete exclusion of infected flowers from pollination may promote plant-to-plant bacterial transfer, giving rise to local epidemic bursts.

Author contribution AC, FS and SA conceived the study and designed the experiments; AC, ID and VG performed all the treatments, samplings and field experiments; MTRE, SS and VG performed the analyses of volatile compounds; BF performed the statistical analysis; FS supervised the whole work. AC and VG drafted the first version of the manuscript. All the authors elaborated the results, actively contributed to their discussion, revised the manuscript and approved the final version.

\section{Compliance with ethical standards}

Conflict of interest The authors declare that they have no conflict of interest.

\section{References}

1. Garratt MP, Breeze TD, Jenner N, Polce C, Biesmeijer JC, Potts SG. Avoiding a bad apple: insect pollination enhances fruit quality and economic value. Agric Ecosyst Environ. 2014;184:34-40.

2. Clarke D, Whitney H, Sutton G, Robert D. Detection and learning of floral electric fields by bumblebees. Science. 2013;340:66-69.

3. Giurfa M, Lehrer M. Honeybee vision and floral displays: from detection to close-up recognition. In: Lars JD, Chittka L, (eds). Cognitive Ecology of Pollination.. Cambridge, UK: Cambridge University Press; 2001. p. 61-82.

4. Harrap MJM, Rands SA, de Ibarra NH, Whitney HM. The diversity of floral temperature patterns, and their use by pollinators. eLife. 2017;6:e31262 https://doi.org/10.7554/eLife.31262

5. Riffell JA. The neuroecology of a pollinator's buffet: olfactory preferences and learning in insect pollinators. Integr Comp Biol. 2011;51:781-93.

6. Farré-Armengol G, Filella I, Llusia J, Peñuelas J. Floral volatile organic compounds: between attraction and deterrence of visitors under global change. Perspect Plant Ecol Syst. 2013;15:56-67.

7. Nepi M, Grasso DA, Mancuso S. Nectar in plant-insect mutualistic relationships: from food reward to partner manipulation. Front Plant Sci. 2018;9:1063 https://doi.org/10.3389/fpls.2018.01063

8. McArt SH, Koch H, Irwin RE, Adler LS. Arranging the bouquet of disease: floral traits and the transmission of plant and animal pathogens. Ecol Lett. 2014;17:624-36.

9. Eigenbrode SD, Bosque-Perez NA, Davis TS. Insect-borne plant pathogens and their vectors: ecology, evolution, and complex interactions. Annu Rev Entomol. 2018;63:169-91.

10. Raguso RA, Roy BA. 'Floral' scent production by Puccinia rust fungi that mimic flowers. Mol Ecol. 1998;7:1127-36.

11. Naef A, Roy BA, Kaiser R, Honegger R. Insect-mediated reproduction of systemic infections by Puccinia arrhenatheri on Berberis vulgaris. New Phytol. 2002;154:717-30.

12. McArt SH, Miles TD, Rodriguez-Saona C, Schilder A, Adler LS, Grieshop MJ. Floral scent mimicry and vector-pathogen associations in a pseudoflower-inducing plant pathogen system. PLoS One. 2016;11:e165761.

13. Dötterl S, Jürgens A, Wolfe L, Biere A. Disease status and population origin effects on floral scent: potential consequences for oviposition and fruit predation in a complex interaction between a plant, fungus, and noctuid moth. J Chem Ecol. 2009;35:307-19.

14. Donati I, Cellini C, Buriani G, Mauri M, Kay C, Tacconi G, et al. Pathways of flower infection and pollen-mediated dispersion of Pseudomonas syringae pv. actinidiae, the causal agent of 
kiwifruit bacterial canker. Hort Res. 2018;5:56 https://doi.org/10. 1038/s41438-018-0058-6

15. Farkas A, Mihalik E, Dorgai L, Buban T. Floral traits affecting fire blight infection and management. Trees. 2011;26:1-20.

16. Eden-Green SJ, Billing E. Fireblight. Rev Plant Pathol. 1974;53:353-65.

17. Wilson M, Lindow SE. Interactions between the biological control agent Pseudomonas fluorescens strain A506 and Erwinia amylovora in pear blossom. Phytopathology. 1993;83:117-23.

18. Johnson KB, Stockwell VO. Biological control of fire blight. In: Vanneste JL, (ed). Fire Blight: the Disease and its Causative Agent. Wallingford, Oxon, UK: Erwinia amylovora, CAB International; 2000. p. 319-38.

19. Vanneste JL, (ed). Fire Blight, the Disease and its Causative Agent Erwinia amylovora. Wallingford, UK: CABI Publishing; 2000.

20. Pusey PL. Effect of nectar on microbial antagonists evaluated for use in control of fire blight of pome fruits. Phytopathology. 1999;89:39-46.

21. Buban T, Orosz-Kovacs Z, Farkas A. The nectary as the primary site of infection by Erwinia amylovora (Burr.) Winslow et al:: a mini review. Plant Syst Evol. 2003;238:183-94.

22. Thomson SV. The role of the stigma in fire blight infections. Phytopathology. 1986;76:476-82.

23. Pusey PL. The role of water in epiphytic colonization and infection of pomaceous flowers by Erwinia amylovora. Phytopathology. 2000;90:1352-7.

24. Spinelli F, Ciampolini F, Cresti M, Geider K, Costa G. Influence of stigmatic morphology on flower colonization by Erwinia amylovora and Pantoea agglomerans. Eur J Plant Pathol. 2005;113:395-405.

25. Wilson M, Sigee DC, Epton HAS. Erwinia amylovora infection of hawthorn blossom: I. The anther. J Phytopathol. 1989;127:1-14.

26. Johnson KB, Stockwell VO, Burgett DM, Sugar D, Loper JE. Dispersal of Erwinia amylovora and Pseudomonas fuorescens by honey bees from hives to apple and pear blossoms. Phytopathology. 1993;83:478-84.

27. Alexandrova M, Porrini C, Bazzi C, Carpana E, Bigliardi M, Sabatini AG. Erwinia amylovora longevity in beehives, beehive products and honeybees. Acta Hortic. 2002;590:201-5.

28. Sisterson MS. Effects of insect-vector preference for healthy or infected plants on pathogen spread: insights from a model. J Econ Entomol. 2008;101:1-8.

29. Roosien BK, Gomulkiewicz R, Ingwell LL, Bosque-Pérez NA, Rajabaskar D, Eigenbrode SD. Conditional vector preference aids the spread of plant pathogens: results from a model. Environ Entomol. 2013;42:1299-308.

30. Sharifi R, Ryu C. Sniffing bacterial volatile compounds for healthier plants. Curr Opin Plant Biol. 2018;44:88-97. https://doi. org/10.1016/j.pbi.2018.03.004.

31. Heil M, Ton J. Long-distance signalling in plant defence. Trends Plant Sci. 2008;13:264-72.

32. Cellini A, Buriani G, Rocchi L, Rondelli E, Savioli S, Rodriguez Estrada MT, et al. Biological relevance of volatile organic compounds emitted during the pathogenic interactions between apple plants and Erwinia amylovora. Mol Plant Pathol. 2018;19:13643703.

33. Arimura G, Matsui K, Takabayashi J. Chemical and molecular ecology of herbivore-induced plant volatiles: proximate factors and their ultimate functions. Plant Cell Physiol. 2009;50:911-23.

34. Junker RR, Loewell C, Gross R, Dötterl S, Keller A, Blüthgen N. Composition of epiphytic bacterial communities differs on petals and leaves. Plant Biol. 2011;13:918-24.

35. Huang M, Sanchez-Moreiras AM, Abel C, Sohrabi R, Lee S, Gershenzon J, et al. The major volatile organic compound emitted from Arabidopsis thaliana flowers, the sesquiterpene $(E)-\beta$ - caryophyllene, is a defense against a bacterial pathogen. New Phytol. 2012;193:997-1008.

36. Velasco R, Zharkikh A, Affourtit J, Dhingra A, Cestaro A, Kalyanaraman A, et al. The genome of the domesticated apple (Malus $\times$ domestica Borkh.). Nat Genet. 2010;42:833.

37. Cornille A, Giraud T, Smulders MJ, Roldán-Ruiz I, Gladieux P. The domestication and evolutionary ecology of apples. Trends Genet. 2014;30:57-65.

38. Ramírez F, Davenport TL. Apple pollination: a review. Sci Hortic. 2013;162:188-203.

39. Tholl D, Boland W, Hansel A, Loreto F, Röse US, Schnitzler JP. Practical approaches to plant volatile analysis. Plant J. 2005;45:540-60.

40. Materić D, Bruhn D, Turner C, Morgan G, Mason N, Gauci V. Methods in plant foliar volatile organic compounds research. Appl Plant Sci. 2015;3:1500044.

41. Vanneste JL, Cornish DC, Yu J, Voyle MD. P10c: a new biological control agent for control of fire blight which can be sprayed or distributed using honey bees. Acta Hortic. 2002;590:231-5.

42. Miller TD, Schroth MN. Monitoring the epiphytic population of Erwinia amylovora on pear with a selective medium. Phytopathology. 1972;62:1175-82.

43. Salm H, Geider K. Real-time PCR for detection and quantification of Erwinia amylovora, the causal agent of fireblight. Plant Pathol. 2004;53:602-10.

44. Vanneste JL. Honey bees and epiphytic bacteria to control fire blight, a bacterial disease of apple and pears. Biocontrol News Inf. 1996;17:67-78.

45. Braun-Kiewnick A, Lehmann A, Rezzonico F, Wend C, Smits TH, Duffy B. Development of species-, strain- and antibiotic biosynthesis-specific quantitative PCR assays for Pantoea agglomerans as tools for biocontrol monitoring. J Microbiol Methods. 2012;90:315-20.

46. Bartolozzi F, Bertazza G, Bassi D, Cristoferi G. Simultaneous determination of soluble sugars and organic acids as their trimethylsilyl derivatives in apricot fruits by gas-liquid chromatography. J Chromatogr A. 1997;758:99-107.

47. Van Den Dool H, Kratz PD. A generalization of the retention index system including linear temperature programmed gas-liquid partition chromatography. J Chromatog A. 1963;11:463-71.

48. Pieterse CMJ, Dicke M. Plant interactions with microbes and insects: from molecular mechanisms to ecology. Trends Plant Sci. 2007;12:564-9.

49. Omata A, Yomogida K, Nakamura S, Hashimoto S, Koba S, Furukawa K, et al. Volatile components of apple flowers (1990). Flavour Frag J. 1990;5:19-22.

50. Buchbauer G, Jirovetz L, Wasicky M, Nikiforovt A. Headspace and essential oil analysis of apple flowers. J Agric Food Chem. 1993;41:116-8.

51. Fraternale D, Flamini G, Donata Ricci D, Giomaro G. Flowers volatile profile of a rare red apple tree from Marche region (Italy). J Oleo Sci. 2014;63:1195-201.

52. Giacomuzzi V, Cappellin L, Nones S, Khomenko I, Knight AL, Biasioli F, et al. Diel rhythms in the volatile emission of apple and grape foliage. Phytochemistry. 2017;138:104-15.

53. Blight MM, Le Métayer M, Delègue MHP, Pickett JA, MarionPoll F, Wadhams LJ. Identification of floral volatiles involved in recognition of oilseed rape flowers, Brassica napus by honeybees, Apis mellifera. J Chem Ecol. 1997;23:1715-27.

54. Kobayashi K, Arai M, Tanaka A, Matsuyama S, Honda H, Ohsawa $\mathrm{R}$. Variation in floral scent compounds recognized by honeybees in Brassicaceae crop species. Breed Sci. 2013;62:293-302.

55. Twidle AM, Mas F, Harper AR, Horner RM, Welsh TJ, Suckling DM. Kiwifruit flower odor perception and recognition by honey bees, Apis mellifera. J Agric Food Chem. 2015;63:5597-602. 
56. Laloi $\mathrm{D}$, Bailez $\mathrm{O}$, Blight $\mathrm{MM}$, Roger $\mathrm{B}$, Pham-Delègue $\mathrm{MH}$, Wadhams LJ. Recognition of complex odors by restrained and freeflying honeybees. Apis mellifera J Chem Ecol. 2000;26:2307-19.

57. Knauer AC, Schiestl FP. Bees use honest floral signals as indicators of reward when visiting flowers. Ecol Lett. 2015;18:135-43.

58. Spaethe J, Tautz J, Chittka L. Do honeybees detect color targets using serial or parallel visual search? J Exp Biol. 2006;209:987-93.24.

59. Wright GA, Smith BH. Variation in complex olfactory stimuli and its influence on odour recognition. Proc R Soc Lond B Biol Sci. 2004;271:147-52.

60. Wright GA, Kottcamp S, Thomson MGA. Generalization mediates sensitivity to complex odor features in the honeybee. PLoS One. 2008;3:e1704 https://doi.org/10.1371/journal.pone.0001704

61. Henning JA, Peng YS, Montague MA, Teuber LR. Honey bee (Hymenoptera: Apidae) behavioral response to primary alfalfa (Rosales: Fabaceae) floral volatiles. J Econ Entomol. 1992;85:233-9.

62. Thiery D, Bluet JM, Pham-Delègue MH, Etiévant P, Masson C. Sunflower aroma detection by the honeybee. J Chem Ecol. 1990;16:701-11.
63. Shulaev V, Silverman P, Raskin I. Airborne signalling by methyl salicylate in plant pathogen resistance. Nature. 1997;385: 718-21.

64. Farag MA, Fokar M, Abd H, Zhang H, Allen RD, Paré PW. Z)-3Hexenol induces defense genes and downstream metabolites in maize. Planta. 2005;220:900-9.

65. Wei J, Kang L. Roles of (Z)-3-hexenol in plant-insect interactions. Plant Signal Behav. 2011;6:369-71.

66. Brown SP, Cornforth DM, Mideo N. Evolution of virulence in opportunistic pathogens: generalism, plasticity, and control. Trends Microbiol. 2012;20:336-42.

67. Maccagnani B, Giacomello F, Fanti M, Gobbin D, Maini S, Angeli G. Apis mellifera and Osmia cornuta as carriers for the secondary spread of Bacillus subtilis on apple flowers. Biocontrol. 2009;54:123-33.

68. Mommaerts V, Smagghe G. Entomovectoring in plant protection. Arthropod-Plant Interact. 2011;5:81-95.

69. Cabrefiga J, Montesinos E. Analysis of aggressiveness of Erwinia amylovora using disease-dose and time relationships. Phytopathology. 2005;95:1430-7. 